\title{
Effect of multipath fading channel in MC-MC-CDMA communication system
}

\author{
Aboura Lamia Touria, Djebbari Ali \\ Telecommunications and Digital Signal Processing Laboratory/ \\ Djillali Liabès University of Sidi Bel-Abbès, Algeria
}

\begin{abstract}
Multi Code Multi Carrier CDMA (MC-MC-CDMA) is the combination of multiple access technique, CDMA, multi code and the OFDM technique. This system is suitable for high rate transmission and multirate services as required in the fourth generation wireless cellular system $(4 G)$. In this paper, the analysis of the effect of the multipath Nakagami fading channel with different multipath intensity profile (MIP) on MC-MCCDMA system is presented. The SNIR (Signal-to-Noise plus Interferences Ratio) and the bit error rate (BER) performance are derived.
\end{abstract}

Keywords-fading parameter, MC-MC-CDMA system, MIP, Nakagami fading channel

\section{INTRODUCTION}

During recent years, wireless communication grows quickly, and it is now capable of the transmission of voice, data and image signals which requires high bandwidth efficiency and high data rates. A new system named MC-MC-CDMA have been suggested for future generation system ; This system makes use advantages of multi code-CDMA technical [1]-[3] and multi carrier modulation with multiple access schema MC-CDMA [4]-[8].

Different forms of MC-MC-CDMA have been described by various authors [9]-[14]. In the system proposed by [9], an M-ary symbol selects one of $\mathrm{M}$ code sequences for transmission. Each chip of the code sequence is copied onto $P$ branches and for the user-specific sequence it is then multiplied with the corresponding branch i.e. the $p^{\text {th }}$ chip of the user-specific sequence is multiplied with the $p^{\text {th }}$ branch of the copier. Each of these branches then modulates one of the $P$ orthogonal subcarriers and the results are summed. In [10], another version of MC-MC-CDMA was proposed. In this system, a high data rate stream is split into a number of parallel low rate streams and then the low rate streams are spread by different sequences and added together. The resulting data is then split into a number of parallel low rate streams and each sub stream modulates a different subcarrier before transmission.

However, in mobile communication system, when a signal transmitted over a radio channel, it is subject to reflexion, refraction and diffusion. The communication environment changes quickly and thus introduces more complexities and uncertainties to the channel response.

In this paper, to observe the effect of fading channel on MC-MC-CDMA system, the characteristics of the channel are investigated, especially the effect of the Nakagami fading parameter [15]-[16] due to its ability to model a wider class of fading channel conditions, and the multipath intensity profile (MIP) [13],[17] which is the average received powers of all multipath components, whole model was considered among them the three kind of channel intensity profile namely Gaussian, exponential and uniform MIPs. The rest of the paper is organized as follows: Section II discusses the MC-MC-CDMA system model described by [10]. The channel models which exhibit both fading and multipath distortions were presented in Section III. In Section IV, the Signal-to-Noise plus Interferences Ratio (SNIR) and the bit error rate (BER) performance are derived. Numerical results are presented in Section V. Finally, Section VI summarized the conclusion had obtained through this paper.

\section{SYSTEM MODEL}

The system model is depicted in Fig.1. It consists of transmitter, receiver and fading channel. The transmitter part converts input data stream $d_{k}(t)$ of $k$ th user, into $J$ parallel substreams $d_{k j}(t)$ which is coded by an orthogonal signal $a_{j}(t)$. The resulting signal $b_{k}(t)$, called superstream, is serial-to-parallel (S/P) converted again and spread in frequency domain by $c_{k}(t)$. Each of these branches then modulates one of the $H$ orthogonal subcarriers and the results are summed. 


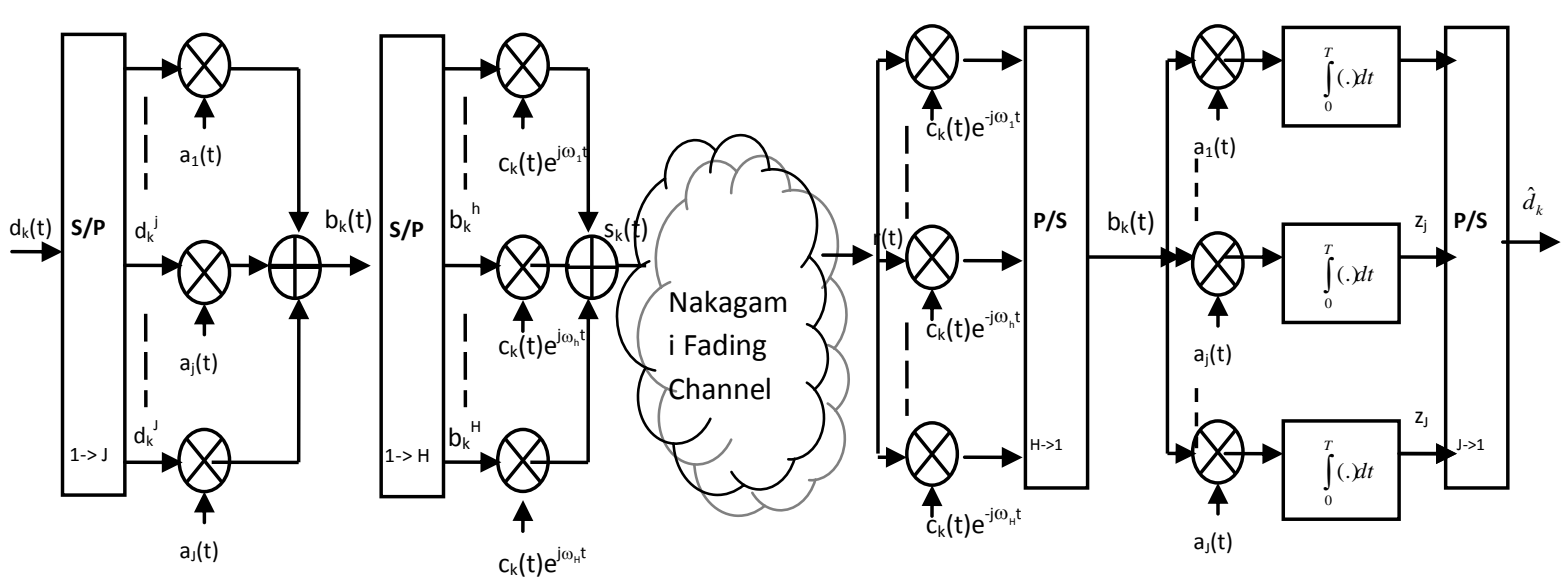

Fig1. Transmitter/Receiver system model for MC-MC-CDMA

At the receiver part, the received signal $r(t)$ is first demodulated by locally generated carrier, despread by the PN sequence and then P/S converted; his output is then despread again by each orthogonal code for multicode component in order to recover substream before correlation. Finally, the substreams are recovered from the correlated data.

To formalize this analysis, assuming that there are $K$ users simultaneously transmitting information, with equal $H, J$ and power $P$; the transmitted signal of the $k$ th user is given by [10]

$$
s_{k}(t)=\sqrt{2 \pi} \sum_{h=1}^{H} \sum_{j=1}^{J} R e\left[d_{k j h}(t) a_{j}(t) c_{k}(t) e^{j \omega_{h} t}\right]
$$

where

$d_{k j h}(t)$ is the data symbol of the $j$ th substream of the $h$ th superstream, consisting in the inphase $d_{k}^{I}(t)$ and quadrature $d_{k}^{Q}(t)$ component, as

$$
d(t)=d_{k}^{I}(t)-j d_{k}^{Q}(t)
$$

$a_{j}(t)$ is the orthogonal signal code for the $j$ th substream which is given by [10]

$$
a_{j}(t)=\sum_{i=0}^{N_{a}-1} a_{j}^{i} \Pi_{\mathrm{T}_{\mathrm{a}}}\left(t-i T_{a}\right)
$$

with $T_{a}=\frac{T}{H \cdot N_{a}}$ is the chip duration of the code, $N_{a}$ is its length and $a_{j}^{i}$ is the $i$ th value of the code $a_{j} \in\{ \pm 1\}$.

The superstream $b_{k}(t)$ is the result of the coding, can written as

$$
b_{k}(t)=\sum_{j=1}^{J} a_{j}(t) d_{k j}(t)
$$

$c_{k}(t)$ is the PN spreading code and is given by

$$
c_{k}(t)=\sum_{i=0}^{N_{c}-1} c_{k}^{i} \Pi_{\mathrm{T}_{\mathrm{c}}}\left(t-i T_{c}\right), \quad T_{c}=\frac{T}{N_{c}}
$$

where $c_{k}^{i}$ is the $i$ th bit value of the code and $N_{c}$ is its length. Notice that the spreading code on all the subcarriers is the same for one particular user.

A transmitted wave undergoes multipath fading which is due to multipath reflections by local dispersers such as man-made structures as buildings, or natural objects such as forest surrounding a mobile unit. Through the multipath fading channel with $L$ paths, the received signal $r(t)$ for all $K$ users is given by [10] as 


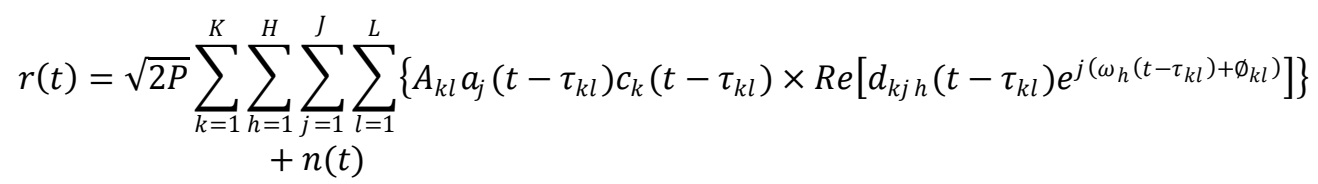

where $\tau_{k l}$ and $\Phi_{k l}$ are the path time and the path phase, respectively. $n(t)$ is the additive white Gaussian noise (AWGN) with a power spectral density of $N_{0} / 2$.

For convenience and yet no loss of generality, we assume that the signal for the first user, first carrier, first orthogonal code via the first path is considered as the reference. In this case [12], $r(t)$ can be written according six components as follows

$$
r(t)=r_{D S}(t)+r_{M P I}(t)+r_{I S S I}(t)+r_{I C I}(t)+r_{M U I}(t)+n(t)
$$

where

$r_{D S}(t)$ is the desired signal, corresponding $k=h=j=l=1$;

$r_{M P I}(t)$ is the MultiPath Interferences caused by the propagation of the desired signal, $k=h=j=1$; via all path except the first path.

$r_{I S S I}(t)$ is the Inter SubStream Interferences caused by other substream except the first substream, $j=1$.

$r_{I C I}(t)$ is the Inter Carriers Interferences caused by all other carriers other than the desired, $h=1$ for the first user.

$r_{M U I}(t)$ is the MultiUser Interferences, caused by all other users except the first user, $k=1$.

Assuming synchronous detection $\left(\tau_{l l}=\phi_{I I}=0\right)$; the output for the correlator for $k=h=j=l=1$ is given by

$$
z_{1}(t)=\int_{0}^{T} r(t) a_{1}(t) c_{1}(t)\left[\cos \left(\omega_{1} t\right)-j \sin \left(\omega_{1} t\right)\right] d t
$$

It is shown in [11] that the output component are

$$
\begin{aligned}
& z_{D S}(t)=\sqrt{\frac{P}{2}} T A_{11}\left[d_{111}^{I}(t)-j d_{111}^{Q}(t)\right] \\
& z_{M P I}(t)=\sqrt{\frac{P}{2}} \sum_{l=2}^{L} A_{1 l} \int_{0}^{T} a_{1}\left(t-\tau_{1 l}\right) a_{1}(t) c_{1}\left(t-\tau_{1 l}\right) c_{1}(t) \\
& \times\left\{d_{111}^{I}\left(t-\tau_{1 l}\right) \cos \left(\theta_{1 l}\right)\right. \\
& +d_{111}^{Q}\left(t-\tau_{1 l}\right) \sin \left(\theta_{1 l}\right)+j d_{111}^{I}\left(t-\tau_{1 l}\right) \sin \left(\theta_{1 l}\right) \\
& \left.-j d_{111}^{Q}\left(t-\tau_{1 l}\right) \cos \left(\theta_{1 l}\right)\right\} d t \\
& Z_{I S S I}(t)=\sqrt{\frac{P}{2}} \sum_{j=2}^{J} \sum_{l=1}^{L} A_{1 l} \int_{0}^{T} a_{j}\left(t-\tau_{1 l}\right) a_{1}(t) c_{1}\left(t-\tau_{1 l}\right) c_{1}(t) \\
& \times\left\{d_{1 j 1}^{I}\left(t-\tau_{1 l}\right) \cos \left(\theta_{1 l}\right)\right. \\
& +d_{1 j 1}^{Q}\left(t-\tau_{1 l}\right) \sin \left(\theta_{1 l}\right)+j d_{1 j 1}^{I}\left(t-\tau_{1 l}\right) \sin \left(\theta_{1 l}\right) \\
& \left.-j d_{1 j 1}^{Q}\left(t-\tau_{1 l}\right) \cos \left(\theta_{1 l}\right)\right\} d t \\
& z_{I C I}(t)=\sqrt{\frac{P}{2}} \sum_{h=2}^{H} \sum_{j=1}^{J} \sum_{l=1}^{L} A_{1 l} \int_{0}^{T} a_{j}\left(t-\tau_{1 l}\right) a_{1}(t) c_{1}\left(t-\tau_{1 l}\right) c_{1}(t) \\
& \times\left\{\operatorname { c o s } ( \theta _ { 1 l } ) \left[d_{1 j h}^{I}\left(t-\tau_{1 l}\right) \cos \left(\omega_{h}-\omega_{1}\right) t\right.\right. \\
& \left.+d_{1 j h}^{Q}\left(t-\tau_{1 l}\right) \sin \left(\omega_{h}-\omega_{1}\right) t\right] \\
& +\sin \left(\theta_{1 l}\right)\left[d_{1 j h}^{Q}\left(t-\tau_{1 l}\right) \cos \left(\omega_{h}-\omega_{1}\right) t\right. \\
& \left.-d_{1 j h}^{I}\left(t-\tau_{1 l}\right) \sin \left(\omega_{h}-\omega_{1}\right) t\right] \\
& +j \cos \left(\theta_{1 l}\right)\left[d_{1 j h}^{Q}\left(t-\tau_{1 l}\right) \cos \left(\omega_{h}-\omega_{1}\right) t\right. \\
& \left.-d_{1 j h}^{I}\left(t-\tau_{1 l}\right) \sin \left(\omega_{h}-\omega_{1}\right) t\right] \\
& +j \sin \left(\theta_{1 l}\right)\left[d_{1 j h}^{I}\left(t-\tau_{1 l}\right) \cos \left(\omega_{h}-\omega_{1}\right) t\right. \\
& \left.\left.+d_{1 j h}^{Q}\left(t-\tau_{1 l}\right) \sin \left(\omega_{h}-\omega_{1}\right) t\right]\right\} d t
\end{aligned}
$$




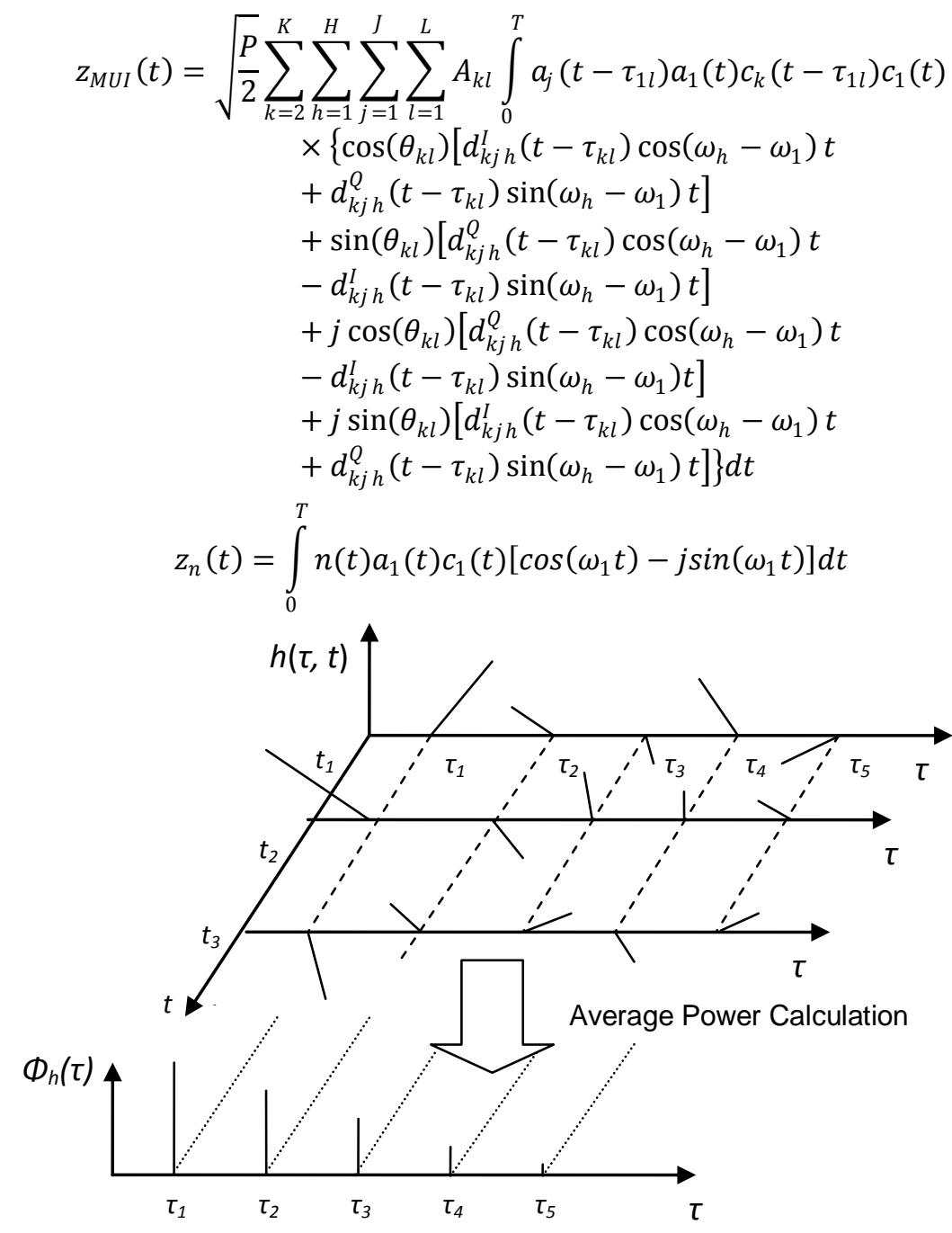

Fig.2. Relationship between MIP and $h(t)$ [5]

\section{ChanNel MOdel}

The channel is considered as conventional multipath channel with equivalent transfer function, $h(t)$ given by [18]

$$
h(t)=\sum_{l=0}^{L-1} A_{k l} e^{j \emptyset_{k l}} \delta\left(t-\tau_{k l}\right)
$$

where $A_{k l}$ is the path gain of $l$ th path of the $k$ th user; The path time delay $\tau_{k l}$ is uniformly distributed over $[0, T]$ and the path phase $\Phi_{k l}$ is uniformly distributed over $[0,2 \pi]$. [12],[18]

The channel path gain $A_{k l}$ has a Nakagami distribution with probability density function (pdf) given by

$$
f_{A_{k l}}\left(A_{k l}\right)=\frac{2}{\Gamma(m)}\left(\frac{m}{\Omega}\right)^{m} A_{k l}^{2 m-1} e^{-\left(\frac{m A_{k l}^{2}}{\Omega}\right)}
$$

where $\Gamma(m)$ is the gamma function and $\Omega$ is the second moment of random variable $A_{k l}$ and $m$ is the Nakagami fading parameter defined as: $\quad m=\frac{\Omega^{2}}{E\left[\left(A_{k l}^{2}-\Omega\right)^{2}\right]}, m \geq 0.5$

The final characteristic which completes our channel model is the multipath intensity profile (MIP) which is the average power output of the channel as a function of the time delay $\tau$; Fig.2 [5] shows the graphical relationship of multipath intensity profile and channel impulse response.

If the average received powers of multipath with equidistant delays are exponentially decaying, then this kind of profile is called an exponentially decaying profile [18] and if they are a Gaussian decaying, then this 
profile is called a Gaussian MIP but if the average received powers of multipath with equidistant delays, are all the same, then it is called independent and identically distributed (i.i.d.) profile or uniform MIP [19].

IV.

SYSTEM ANALYSIS

This section provides results for the analysis of our system. In the following, we concentrate on the performance of MC-MC-CDMA system in terms of the Signal-to-Noise plus Interferences ratio (SNIR), which is the ratio of signal power to noise plus interference variance.

The SNIR is defined in [11] assuming that all terms are zero means, statistically independent random variables. It is expressed as:

$$
\gamma=\frac{\frac{P}{2} A_{11}^{2} T^{2}}{\sigma_{T}^{2}}=A_{11}^{2} R
$$

where $R=\frac{\frac{P T^{2}}{2}}{\sigma_{T}^{2}}$ and $\sigma_{T}^{2}$ is the totally variance, given by [11]

$$
\begin{gathered}
\sigma_{T}^{2}=\frac{P T^{2}}{2}\left\{\frac{1}{3 J H N_{c}} \sum_{l=2}^{L} \operatorname{var}\left[A_{1 l}\right]+\frac{J-1}{3 J H N_{c}} \sum_{l=1}^{L} \operatorname{var}\left[A_{1 l}\right]+\left\{\sum_{h=2}^{H} \frac{J\left[F^{c}(h)-F^{S}(h)\right]}{4 \pi^{2} J^{2}(h-1)^{2} N_{1}}\right\} \sum_{l=1}^{L} \operatorname{var}\left[A_{1 l}\right]\right. \\
\left.+\left\{\frac{J(K-1)}{3 J H N_{c}}+\sum_{h=1}^{H} \frac{J(K-1)\left[F^{c}(h)-F^{S}(h)\right]}{4 \pi^{2} J^{2}(h-1)^{2} N_{1}}\right\} \times \sum_{l=1}^{L} \operatorname{var}\left[A_{k l}\right]+\frac{1}{2 \frac{E_{b}}{N_{0}}}\right\}
\end{gathered}
$$

where $E_{b}=P T$ and $E_{b} / N_{o}$ is the signal-to-noise ratio (SNR).

$F^{c}(h)$ and $F^{s}(h)$ are defined in [11] as follows:

$$
\begin{aligned}
F^{c}(h)=N_{c}\left\{\frac{1}{N_{c}}-\right. & \frac{1}{2 N_{c}} \cos \left(4 \pi \frac{J(h-1)}{T} g T_{c}\right) \\
& +\frac{1}{\pi J(h-1)} \sin \left(2 \pi \frac{J(h-1)}{T} g T_{c}\right) \cos \left(2 \pi \frac{J(h-1)}{T}(g+1) T_{c}\right) \\
& -\frac{1}{2 \pi J(h-1)} \sin \left(2 \pi \frac{J(h-1)}{T} g T_{c}\right) \cos \left(2 \pi \frac{J(h-1)}{T} g T_{c}\right)+\frac{1}{N_{c}} \\
& -\frac{1}{2 N_{c}} \cos \left(4 \pi \frac{J(h-1)}{T}(g+1) T_{c}\right) \\
& +\frac{1}{2 \pi J(h-1)} \sin \left(2 \pi \frac{J(h-1)}{T}(g+1) T_{c}\right) \cos \left(2 \pi \frac{J(h-1)}{T}(g+1) T_{c}\right) \\
& \left.-\frac{1}{\pi J(h-1)} \sin \left(2 \pi \frac{J(h-1)}{T}(g+1) T_{c}\right) \cos \left(2 \pi \frac{J(h-1)}{T} g T_{c}\right)\right\} \\
F^{s}(h)=N_{c}\left(\frac{1}{N_{c}}+\right. & \frac{1}{2 N_{c}} \cos \left(4 \pi \frac{J(h-1)}{T} g T_{c}\right)+\frac{1}{4 \pi J(h-1)} \sin \left(4 \pi \frac{J(h-1)}{T}(g+1) T_{c}\right) \\
& -\frac{1}{\pi J(h-1)} \sin \left(2 \pi \frac{J(h-1)}{T}(g+1) T_{c}\right) \cos \left(2 \pi \frac{J(h-1)}{T} g T_{c}\right) \\
& +\frac{1}{2 \pi J(h-1)} \sin \left(2 \pi \frac{J(h-1)}{T} g T_{c}\right) \cos \left(2 \pi \frac{J(h-1)}{T} g T_{c}\right)+\frac{1}{N_{c}} \\
& +\frac{1}{2 N_{c}} \cos \left(4 \pi \frac{J(h-1)}{T}(g+1) T_{c}\right) \\
& -\frac{1}{\pi J(h-1)} \cos \left(2 \pi \frac{J(h-1)}{T}(g+1) T_{c}\right) \sin \left(2 \pi \frac{J(h-1)}{T}(g+1) T_{c}\right) \\
& \left.+\frac{1}{\pi J(h-1)} \cos \left(2 \pi \frac{J(h-1)}{T}(g+1) T_{c}\right) \sin \left(2 \pi \frac{J(h-1)}{T} g T_{c}\right)\right\}
\end{aligned}
$$

Now, we need to calculate the variance of the path gain $A_{k l}$; this value depends on the nature of the MIP. It can be exponential, uniform or Gaussian; these values of MIPs are shown below.

1. Exponential MIP

For this profile, the different amplitude levels of different paths have exponential relation with the first arrived signal component [13]. Assume amplitude decaying factor of $\delta$ and the path gains are independent and identically distributed random variable, then we can write 


$$
\operatorname{var}\left[\sum_{l=1}^{L} A_{k l}\right]=\sum_{l=1}^{L} \operatorname{var}\left[A_{k l}\right]=\sum_{l=1}^{L} \operatorname{var}\left[A_{k 1} e^{-\delta(l-1)}\right]=\operatorname{var}\left[A_{k 1}\right] \sum_{l=1}^{L} e^{-2 \delta(l-1)}=\Omega Q_{e}(L, \sigma)
$$

where $\Omega$ is the variance of $A_{k l}$ and $Q_{e}$ is the exponential MIP given by

$$
Q_{e}(L, \sigma)=\sum_{l=1}^{L} e^{-2 \delta(l-1)}=1+e^{-2 \delta}+e^{-4 \delta}+\cdots+e^{-2(L-1) \delta}=\frac{1-e^{-2 \delta L}}{1-e^{-2 \delta}}
$$

2. Gaussian MIP

In this case [13], the successive amplitude levels will follow a Gaussian distribution. Then

$$
\operatorname{var}\left[\sum_{l=1}^{L} A_{k l}\right]=\operatorname{var}\left[A_{k 1}\right] \sum_{l=1}^{L} \frac{1}{2 \pi \sigma^{2}} e^{-2(l-1-\mu)^{2} / 2 \sigma^{2}}=\Omega Q_{g}(L, \mu, \sigma)
$$

where $\mu$ and $\sigma$ are the mean and variance of the distribution, respectively. $Q_{g}$ denotes the Gaussian MIP given by

$Q_{g}(L, \mu, \sigma)=\sum_{l=1}^{L} \frac{1}{2 \pi \sigma^{2}} e^{-(l-1-\mu)^{2} / \sigma^{2}}=\frac{1}{2 \pi \sigma^{2}}\left\{e^{-\mu^{2} / \sigma^{2}}+e^{-(1-\mu)^{2} / \sigma^{2}}+\cdots+e^{-(L-1-\mu)^{2} / \sigma^{2}}\right\}$

3. Uniform MIP

In the uniform MIP [13], the amplitude levels of all multipath components are the same. Thus

$$
\operatorname{var}\left[\sum_{l=1}^{L} A_{k l}\right]=\sum_{l=1}^{L} \operatorname{var}\left[A_{k l}\right]=\operatorname{var}\left[A_{k 1}\right] \sum_{l=1}^{L} 1=\Omega Q_{u}
$$

where $Q_{u}=L$ is the uniform MIP.

Thus, we can write SNIR as

$$
\begin{aligned}
\gamma=A_{11}{ }^{2}\left\{\frac{1}{3 J H N_{c}}\right. & \Omega(Q-1)+\frac{J-1}{3 J H N_{c}} \Omega Q+\left\{\sum_{h=2}^{H} \frac{J\left[F^{c}(h)-F^{s}(h)\right]}{4 \pi^{2} J^{2}(h-1)^{2} N_{1}}\right\} \Omega Q \\
& \left.+\left\{\frac{J(K-1)}{3 J H N_{c}}+\sum_{h=1}^{H} \frac{J(k-1)\left[F^{c}(h)-F^{s}(h)\right]}{4 \pi^{2} J^{2}(h-1)^{2} N_{1}}\right\} \Omega Q+\frac{1}{2 \frac{E_{b}}{N_{0}}}\right\}^{-1}
\end{aligned}
$$

where $Q$ may represent $Q_{e}, Q_{g}$ or $Q_{u}$.

To compare the performance of MC-MC-CDMA system, the SNIR of MC-CDMA and MultiCode CDMA was also established following the same steps. We found:

The SNIR of MC-CDMA system is:

$$
\begin{aligned}
\gamma & =A_{11}^{2}\left\{\frac{1}{3 H N_{c}} \Omega(Q-1)+\left\{\sum_{h=2}^{H} \frac{\left[G^{c}(h)-G^{s}(h)\right]}{4 \pi^{2}(h-1)^{2} N_{1}}\right\} \Omega Q\right. \\
& \left.+(K-1)\left\{\frac{1}{3 H N_{c}}+\sum_{h=1}^{H} \frac{\left[G^{c}(h)-G^{s}(h)\right]}{4 \pi^{2}(h-1)^{2} N_{1}}\right\} \Omega Q+\frac{1}{2 \frac{E_{b}}{N_{0}}}\right\}^{-1}
\end{aligned}
$$

with $G^{c}(h)$ and $G^{s}(h)$ are given by:

$$
\begin{aligned}
G^{c}(h)=N_{c}\left\{\frac{1}{N_{c}}\right. & +\frac{1}{2 N_{c}} \cos \left(4 \pi \frac{(h-1)}{T} g T_{c}\right) \\
& +\frac{1}{\pi(h-1)} \sin \left(2 \pi \frac{(h-1)}{T} g T_{c}\right) \cos \left(2 \pi \frac{(h-1)}{T}(g+1) T_{c}\right) \\
& -\frac{1}{2 \pi(h-1)} \sin \left(2 \pi \frac{(h-1)}{T} g T_{c}\right) \cos \left(2 \pi \frac{(h-1)}{T} g T_{c}\right)+\frac{1}{N_{c}} \\
& -\frac{1}{2 N_{c}} \cos \left(4 \pi \frac{(h-1)}{T}(g+1) T_{c}\right) \\
& +\frac{1}{2 \pi(h-1)} \sin \left(2 \pi \frac{(h-1)}{T}(g+1) T_{c}\right) \cos \left(2 \pi \frac{(h-1)}{T}(g+1) T_{c}\right) \\
& \left.-\frac{1}{\pi(h-1)} \sin \left(2 \pi \frac{(h-1)}{T}(g+1) T_{c}\right) \cos \left(2 \pi \frac{(h-1)}{T} g T_{c}\right)\right\}
\end{aligned}
$$




$$
\begin{aligned}
G^{s}(h)=N_{c}\left\{\frac{1}{N_{c}}\right. & +\frac{1}{2 N_{c}} \cos \left(4 \pi \frac{(h-1)}{T} g T_{c}\right)+\frac{1}{4(h-1)} \sin \left(4 \pi \frac{(h-1)}{T}(g+1) T_{c}\right) \\
& -\frac{1}{\pi(h-1)} \sin \left(2 \pi \frac{(h-1)}{T}(g+1) T_{c}\right) \cos \left(2 \pi \frac{(h-1)}{T} g T_{c}\right) \\
& +\frac{1}{2 \pi(h-1)} \sin \left(2 \pi \frac{(h-1)}{T} g T_{c}\right) \cos \left(2 \pi \frac{(h-1)}{T} g T_{c}\right)+\frac{1}{N_{c}} \\
& +\frac{1}{2 N_{c}} \cos \left(4 \pi \frac{(h-1)}{T}(g+1) T_{c}\right) \\
& -\frac{1}{\pi(h-1)} \cos \left(2 \pi \frac{(h-1)}{T}(g+1) T_{c}\right) \sin \left(2 \pi \frac{(h-1)}{T}(g+1) T_{c}\right) \\
& \left.+\frac{1}{\pi(h-1)} \cos \left(2 \pi \frac{(h-1)}{T}(g+1) T_{c}\right) \sin \left(2 \pi \frac{(h-1)}{T} g T_{c}\right)\right\}
\end{aligned}
$$

The SNIR of Multi Code CDMA is written as:

$$
\gamma=A_{11}^{2}\left\{\frac{1}{3 J N_{c}} \Omega(\mathrm{Q}-1)+\frac{J-1}{3 J N_{c}} \Omega Q+\frac{J(K-1)}{3 J N_{c}} \Omega Q+\frac{1}{2 \frac{E_{b}}{N_{0}}}\right\}^{-1}
$$

The performance of the above systems by the average Bit Error Rate (BER) is given by [14]

$$
\bar{P}_{e}=\int_{0}^{+\infty} f_{\gamma}(\gamma) P_{e}(\gamma) d \gamma
$$

where $f_{\gamma}(\gamma)$ is the pdf of $\gamma$ and $P_{e}(\gamma)$ is the conditional bit error probability of the SNIR $\gamma$ is given by [10]

$$
P_{e}(\gamma)=\frac{1}{2} \operatorname{erfc}(\gamma)
$$

The channel path gain is assumed to be Nakagami distributed random variable, then $\gamma$ is also Nakagami distributed with pdf given by [13]

$$
f_{\gamma}(\gamma)=\frac{1}{\Gamma(m)}\left(\frac{m}{\Omega \mathrm{R}}\right)^{m} \gamma^{m-1} e^{-\left(\frac{m \gamma}{\Omega \mathrm{R}}\right)}
$$

Then the BER become

$$
\bar{P}_{e}=\frac{1}{2 \Gamma(m)}\left(\frac{m}{\Omega \mathrm{R}}\right)^{m} \int_{0}^{+\infty} \gamma^{m-1} e^{-\left(\frac{m \gamma}{\Omega \mathrm{R}}\right)} \operatorname{erfc}(\gamma) d \gamma
$$

\section{NUMERICAL RESULTS}

Having derived the output SNIR and BER, the performance of the system is presented in this section. The number of users, the MIP and parameter fading were considered with the parameters listed in Table 1.

Table1. Simulation parameters

\begin{tabular}{|l|l|}
\hline Parameter Description & Value \\
\hline Total bandwidth & $1 \mathrm{MHz}$ \\
\hline Number of multipath & $L=3$ \\
\hline Number of user substreams & $J=8$ \\
\hline Number of carriers & $H=8$ \\
\hline Local mean power & $\Omega=10 \mathrm{~dB}$ \\
\hline Number of chips per bit & $N_{l}=4$ \\
\hline
\end{tabular}

Fig. 3 Illustrates the SNIR performance of the different system MC-MC-CDMA, MC-CDMA and Multi Code CDMA for BPSK modulation, the figure clearly shows that the MC-MC-CDMA system has the best performance of all the systems. This is due to the gain which comes from the combination of MC-CDMA and Multi Code CDMA. 
Fig.4 shows a plot of the SNIR performance of MC-MC-CDMA system for several values of number of users $K$, for BPSK modulation; For the Rayleigh channel, the fading parameter is taken $m=1$. It is clear from those figures that, as many users are transmitting signal simultaneously, the SNIR decreases; Hence, the higher the number of users, the higher the multiuser interference caused by the unwanted user and consequently the performance become worse.

Fig.5 shows the effect of exponential, uniform and Gaussian MIPs on the SNIR performance of MCMC-CDMA system; we have considered $K=20$ and $m=1$. The MIP performance is affected by the $Q$ parameters, which is the MIP parameter for different intensity profile. For a fair comparison, we assumed for the Gaussian profile that $\mu=0$ and $\sigma^{2}=1 / 2 \pi$, the decay factor of the exponential MIP is taken $\sqrt{2 \pi}$ thus, the $Q$ value for Gaussian MIP is 1.0882 and 1.0067 for exponential MIP and 3 for uniform MIP, which are calculating using (22), (24) and (25). The figure shows that there is no significant difference between exponential and Gaussian MIPs and these profiles have better performance than uniform MIP. Therefore the less of the value of $Q$, the less the variance for interference, the better is SNIR.

Fig. 6 shows the effect of channel fading on BER performance for MC-MC-CDMA system using exponential MIP. We used different fading parameter, $m=0.5$ for the half Gaussian fading, $m=1$ for Rayleigh model and $m=3$ for approximated Rician model. Clearly, the higher value of $m$, the better the performance of the system, which corresponds to a less fading in the channel or the Rician channel which is suitable to model the indoor channel; the half Gaussian model corresponds the worst case fading condition, it is suitable for the urban area.

\section{CONCLUSION}

In this paper, the effect of channel fading in MC-MC-CDMA communication system is analyzed. We investigated how various parameters of channel, such as the MIP and fading parameter, affect the MC-MCCDMA system performance. The results illustrated that the phenomena of channel fading dominate the performance of MC-MC-CDMA communication system. However, the most important factor should be the fading parameter of the fading model.

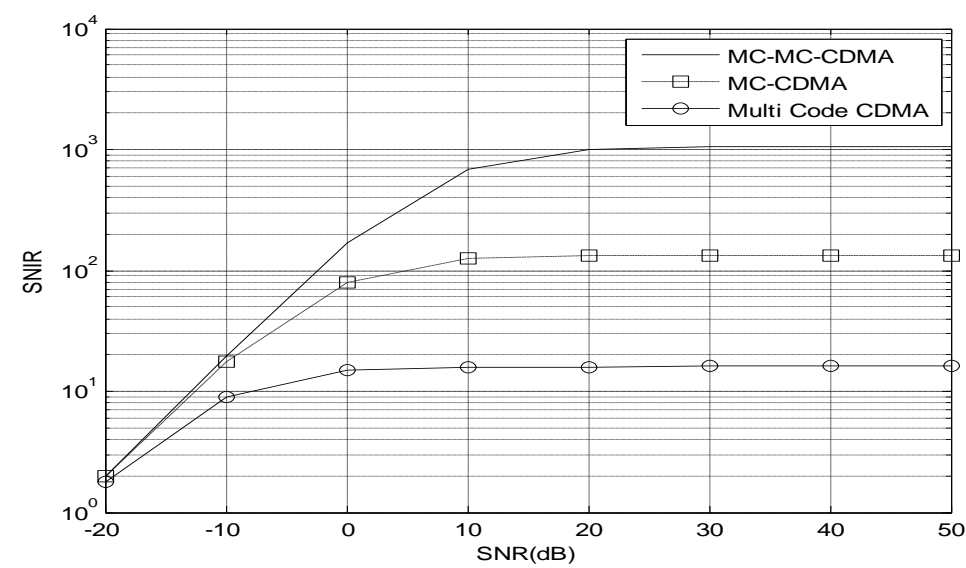

Fig.3. SNIR performance for different CDMA based systems

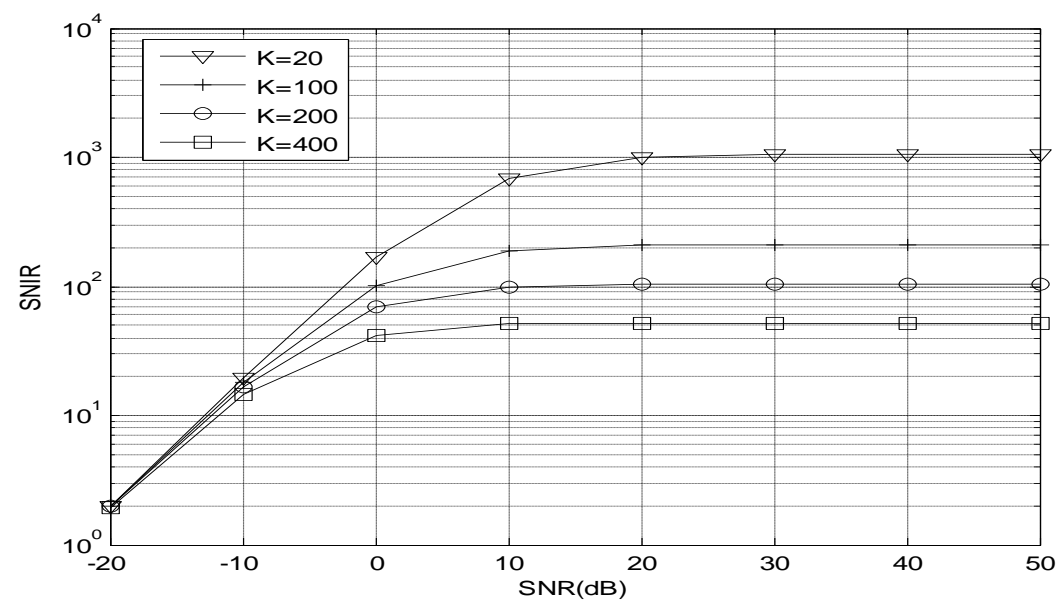

Fig.4. Effect of number of users, $K$, in the MC-MC-CDMA system 


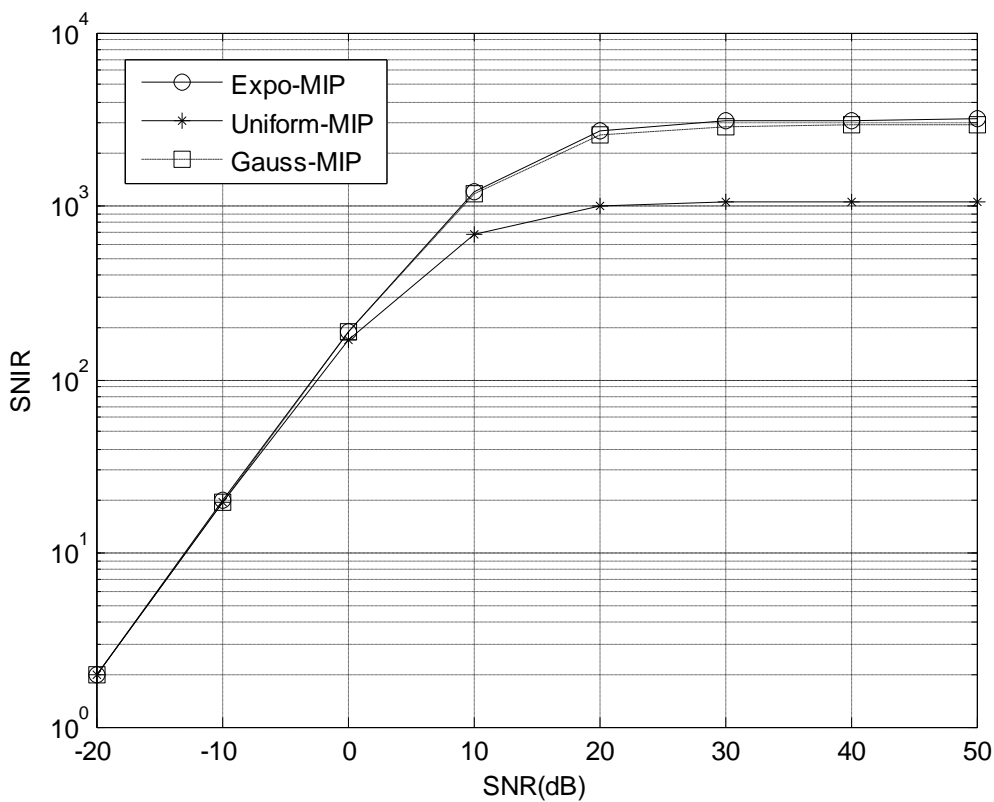

Fig .5. Effect of the MIP on SNIR performance

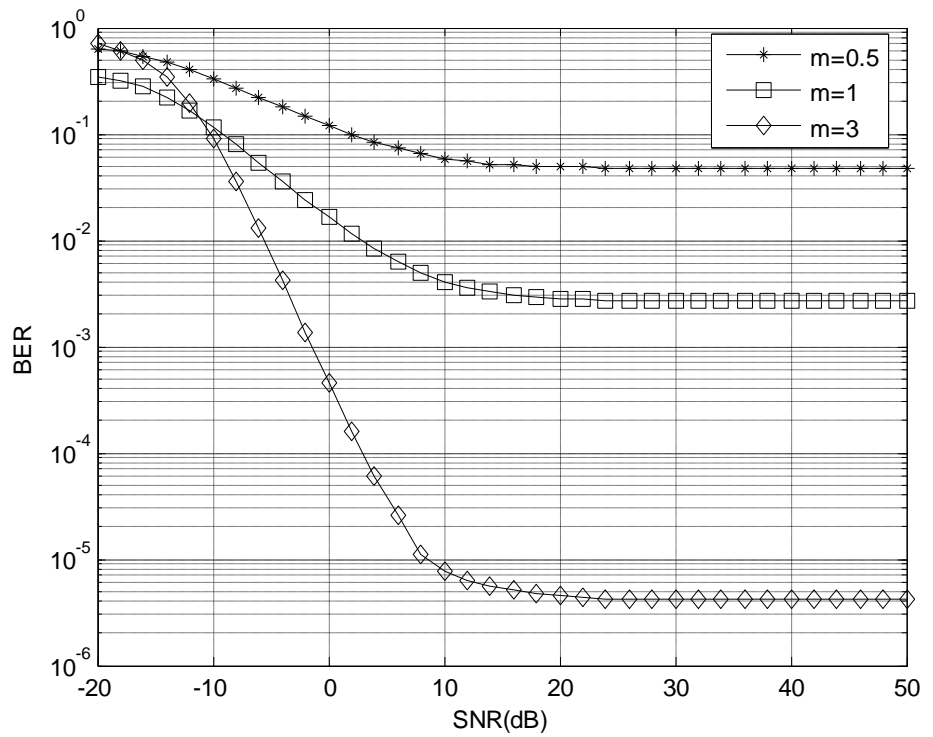

Fig. 6. Effect of fading parameter, $m$, on BER performance

\section{REFERENCES}

[1] M. Kim, B. C. Shin, Y. J. Kim, J. K. Kim and I. Han, Throughput improvement scheme in multicode CDMA, Electronics Letters, vol 34, 1998, pp. 963-964.

[2] D. W. Hsiung and J. F. Chang, Performance of multicode CDMA in a multipath fading channel, IEEE Commun., vol. 147, 2000, pp.365-370

[3] G. V. S. Raju and J.Charoensakwiroj, Orthogonal codes performance in multicode CDMA, 2003 IEEE Int. Conf. on Systems, Man and Cybernetics, vol. 2, 2003, pp. 1928-1931.

[4] S. Hara and R. Prasad, Overview of Multicarrier CDMA, IEEE Commun. vol. 35, no. 12, 1997, pp. 126-133.

[5] S. Hara and R. Prasad, MULTICARRIER TECHNIQUES FOR 4G MOBILE COMMUNICATIONS (Artech House, ISBN: 1-58053482-1: London, 2003).

[6] E. Sourour, M. Nakagawa, Performance of orthogonal multicarrier CDMA in a multipath fading channel, IEEE Trans. Commun., vol. 44,1996, pp. 356-367.

[7] J. H. Park, J. E. Kim, S. Y. Choi, N. S. Cho and D. S. Hong, Performance of MCCDMA systems in non-independent Rayleigh fading, IEEE Int. Commun.Conf., Vancouver, BC Canada, vol. 1, 1999, pp. 06-10.

[8] K. W. Ryu, J. O. Park and Y. W. Park, Performance of multicarrier CS/CDMA in frequency-selective Rayleigh fading channels, 2003 Spring IEEE $57^{\text {th }}$ Semiannual Vehicular Technology Conf., vol. 2, 2003, pp. 1258-1262.

[9] T. Kim, J. Kim, J. G. Andrews, and T. S. Rappaport, Multi-code Multicarrier CDMA: Performance Analysis, in Proc.IEEE Int. Conf. Comm., vol.2, June 2004, pp.973-977. 
[10] J.W. Lee, O.C. Ugweje and C. Madubata, Multi-Code Multi-Carrier systems for 4G wireless communications, Proceedings of the Thirty-Seventh Southeastern Symposium on System Theory, Mar. 2005, pp. 20-24.

[11] L.T. Aboura, A. Djabbari, Derivation of new expression of SNIR for Multi-Code Multi-Carrier CDMA systems, Proceedings of the SPIT-ASP, Dubai, 20-21 September 2012, pp. 219-228.

[12] J.W. Lee, Performance analysis of Multi-code Multi Carrier CDMA communication system, Master Degree Thesis, Univ. Akron, Akron, OH, Dec. 2004.

[13] M.M. Akho-zahieh, Design and analysis of multicarrier multicode wavelet packets based CDMA communication systems with multiuser detection, Ph.D. dissertation, Univ. Akron, Akron, OH, Aug. 2006.

[14] M.M. Akho-zahieh and O.C. Ugweje, Diversity performance of Wavelet-Packet based Multi-Code Multi-Carrier communication systems, IEEE Trans. On vehicular technology, Vol.57, no.8, March 2008, pp. 787-796.

[15] N. C. Beaulieu and C. Cheng, An efficient procedure for Nakagami-m fading simulation. In IEEE Proc. of Globecom 2001, volume 6, Nov 2001, pp. 3336-3342.

[16] K. W. Yip and T. S. Ng, A simulation model for Nakagami-m fading channels, $m<1$. IEEE Transactions on Communications, 48(2), Feb 2000, pp.214-221.

[17] Ghassemzadeh, S.S., Greenstein, L.J., Sveinsson, T. Tarokh, A multipath intensity profile model for residential environments, IEEE Wireless Communications and Networking, vol.1 , 2003, pp.150-155.

[18] H. HASHEMI, The Indoor Radio Propagation Channel, Proc. IEEE, Vol.81 (7), July 1993, pp. 943 - 68.

[19] R. STEELE, Mobile radio communications (Pentech IEEE Press, ISBN: 0-7803-1102-7, New York, 1992). 